\title{
Discours sur la musique à Hérat (Afghanistan)
}

Expert and non-expert discourse about music in Herat, Afganisatan

John Baily

Traducteur : Ramèche Goharian

\section{QpenEdition}

\section{Journals}

Édition électronique

URL : http://journals.openedition.org/ethnomusicologie/1586

ISSN : 2235-7688

\section{Éditeur}

ADEM - Ateliers d'ethnomusicologie

\section{Édition imprimée}

Date de publication : 1 janvier 1998

Pagination : $125-140$

ISBN : 2-8257-0639-6

ISSN : $1662-372 X$

\section{Référence électronique}

John Baily, « Discours sur la musique à Hérat (Afghanistan) », Cahiers d'ethnomusicologie [En ligne], 11 | 1998, mis en ligne le 07 janvier 2012, consulté le 01 mai 2019. URL : http:// journals.openedition.org/ethnomusicologie/1586

Ce document a été généré automatiquement le 1 mai 2019.

Tous droits réservés 


\title{
Discours sur la musique à Hérat (Afghanistan)
}

\author{
Expert and non-expert discourse about music in Herat, Afganisatan
}

John Baily

Traduction : Ramèche Goharian

\section{NOTE DE L'ÉDITEUR}

Traduit de l'anglais

\section{Le concept de « science » à Hérat ${ }^{2}$}

1 A Hérat comme dans une grande partie du monde musulman, les domaines de la connaissance qui inspiraient le respect étaient appelés ilm, «science ». Un ilm n'était pas une science fondée sur une méthode empirique, comme on l'entend en Occident, mais plutôt une somme de connaissances organisées de façon systématique, comportant un ensemble de concepts et de terminologies qui lui étaient propres et relevant de la compétence de praticiens spécialistes ou d'experts. Parmi ces sciences, l'ilm-e tajwid ou «la science de la prononciation correcte du Coran » occupait un rang prééminent. Les musulmans considèrent le Coran comme la parole de Dieu, miraculeusement transmise au prophète Mohammad. La langue du Coran est l'arabe classique et l'on accorde une grande importance à sa prononciation correcte, exigence difficile à satisfaire car l'arabe coranique contient des phonèmes inexistants en arabe moderne ou en persan, la langue parlée à Hérat. En prononçant le texte de façon incorrecte, on risque d'altérer son sens et de créer ainsi une situation inacceptable. L'ilm-e tajwid se compose d'un système phonologique sophistiqué et d'une série complexe de règles, issues de la grammaire arabe, qui contrôlent les divers aspects du qirâ'at, ce terme désignant les différentes formes de la récitation, de la cantillation et du chant coraniques. A Hérat, l'ilm-e tajwid faisait partie du programme d'études des écoles coraniques. Ayant enregistré le qirầat de 
vingt étudiants de théologie, en 1977, j'ai fait écouter ces bandes au professeur Maulawi Nurahmad, un des rares mullahs de Hérat doté d'une solide connaissance du tajwid, qu'il avait étudié en Inde dans la fameuse école de Deoband Madressa. Celui-ci déclara qu'une seule de ces lectures était correcte selon les critères de l'ilm-e tajwid. L'étudiant avait compris l'ilm et appliqué cette connaissance à l'apprentissage du qirấat. Les autres avaient appris machinalement, en écoutant leur professeur réciter et en essayant de l'imiter phrase par phrase.

2 La musique avait, elle aussi, sa science, l'ilm-e musiqi. Ensemble de règles relatives à la connaissance musicale exprimées verbalement, elle était l'apanage des musiciens urbains et, plus particulièrement, des spécialistes de la musique kabouli. Cette science décrivait essentiellement les modes mélodiques et les cycles métriques que les musiciens employaient dans leurs interprétations et s'intéressait surtout à deux éléments fondamentaux de la musique, sor et lai, la « hauteur » et le « rythme ». La science musicale connue à Hérat ressemblait fort à la théorie de la musique hindoustanie dont elle était certainement dérivée, par l'entremise des musiciens de cour indiens amenés à Kaboul à la fin du XIXe siècle et, plus tard, par l'adoption de l'art musical kabouli à Hérat dans les années 1930. Il s'agissait d'une "version" de la théorie hindoustanie qui désignait souvent des notions identiques par des termes similaires, même si certaines divergences pouvaient parfois apparaître. A la base de cette science se trouvait un système de noms de notes, le système sargam, tel qu'il est connu en Asie du Sud (Sa Re Ga Ma Pa Dha Ni Sa). Ces noms de notes pouvaient être reproduits sous une forme écrite, mais ils servaient essentiellement de «notation orale » et de représentation verbale de la hauteur, comme on solfie en musique européenne ${ }^{3}$. Quant à la notation écrite et verbale des cycles métriques, on utilisait le système de syllabes mnémoniques pour tambours appelées tabla bol (Dha, Dhin, Na, Ta, Kat, Dhum, etc.).

3 L'existence d'une telle science de la musique rendait les musiciens et leur art plus respectables, en prouvant que l'exécution musicale avait un fondement rationnel. La possibilité même de discourir sur les éléments structurels de la musique changeait le statut de celle-ci, dans une société islamique traditionnelle qui la considérait avec une certaine méfiance. La tradition hératie d'une science musicale écrite remonte au moins à l'époque du savant, poète et mystique soufi Abdur Rahman Jami, qui publia son traité sur la musique au XV siècle (Beliaev 1960).

\section{Connaissance spécialisée en théorie musicale hindoustanie}

4 L'ilm-e musiqi était une théorie musicale, au sens où l'entend Feld (1981), dans son analyse de la musique kaluli des Papous de Nouvelle-Guinée :

« Le caractère principal d'une théorie réside dans l'organisation systématique de la pensée (ibid. 23)... La théorie musicale consiste en une réflexion systématique sur le phénomène des structures sonores reliées entre elles (ibid. 39)... La théorie musicale... est... un système conceptuel de principes culturels qui structure la connaissance, la production et l'interprétation des sons organisés dans un milieu social... Le son n'est pas seulement produit, il est socialement formulé en tant que théorie » (ibid. 45) ${ }^{4}$.

5 L'ilm-e musiqi était réservé aux musiciens professionnels urbains; les femmes musiciennes, les amateurs ou les non-musiciens y avaient peu accès. Il pouvait y avoir de 
grandes différences dans le degré de connaissance des musiciens spécialisés, qui utilisaient la terminologie de la science musicale pour parler de musique dans divers contextes. Cela faisait partie de leur langage commun et de leur culture spécifique de musiciens. Ils l'employaient lors d'une écoute critique ou pendant des discussions plus générales sur la musique et s'en servaient aussi dans leurs perpétuelles rivalités pour se hisser à un rang plus élevé au sein de leur communauté. Un musicien qui connaissait un point obscur de la théorie musicale comme, par exemple, le terme désignant un râg (mode mélodique) à cinq tons dans ses échelles ascendantes et descendantes ou des détails sur un râg rare, l'emportait toujours sur celui qui les ignorait. Même les meilleurs sâzandeh (musiciens professionnels) de Hérat étaient conscients du fait qu'ils n'en savaient pas autant que les grands ustâd (maîtres) de Kaboul. Ils attachaient un prix énorme à la moindre parcelle de savoir qu'ils pouvaient recueillir et ne transmettaient leurs connaissances que dans le cadre de la relation de maittre à élève (Baily 1988 : 121).

6 L'ilm-e musiqi servait durant la période de formation des jeunes musiciens; mais pour mieux expliquer le rôle joué par le discours sur la musique dans le processus d'apprentissage, il faut entrer ici dans un peu plus de détails. Parmi les emprunts faits à la musique indienne par les musiciens de Hérat, se trouvait le concept de la relation ustâdshâgird, de maître à élève. Cette relation était confirmée lors d'une cérémonie où l'ustâd nouait un cordon de sept couleurs autour du poignet de son shâgird (Baily 1988 : 121-123). Mais, alors qu'elle était présentée comme un idéal, cette relation pédagogique servait peu comme moyen d'instruction; quant à l'ilm-e musiqi, il jouait un rôle négligeable dans la méthode d'enseignement élémentaire. Les jeunes musiciens apprenaient essentiellement par l'oreille, au contact direct des sons musicaux, en imitant les interprétations musicales et par tâtonnement, dans un environnement social où la pratique musicale était vivement encouragée par les membres de leur famille (Baily 1988b : 119 ; Baily and Doubleday 1988) ${ }^{5}$. Les musiciens avaient beau parler de théorie musicale, se vanter de connaître des termes obscurs ou se présenter comme ilmdâr, détenteurs de la science, ils n'avaient pas acquis leur savoir par le biais d'un enseignement fondé sur la théorie musicale. Plus précisément, ils n'apprenaient pas au moyen de la notation. Le jeune musicien qui avait appris son art par le processus imitatif mentionné plus haut reconnaissait, après coup, des noms de notes, de tabla bol, de râg ou de tâl etc. La science musicale décrivait certains principes permettant la structuration de la musique et formulait des distinctions terminologiques grâce auxquelles ces principes pouvaient être exprimés verbalement. Elle témoignait d'une "riche connaissance de base», mais la théorie n'était pas la connaissance elle-même, elle tentait simplement de représenter la connaissance musicale sous une forme verbale, comme une rationalisation a posteriori de la pratique musicale.

\section{Modèles représentatifs et opérationnels de la structure musicale}

7 J'aimerais introduire ici une distinction fondamentale entre les modèles représentatifs et opérationnels de la structure musicale. Bien qu'il s'agisse d'entités cognitives en rapport avec le rôle cognitif de la théorie musicale, ils ressortissent au phénomène de la verbalisation sur la musique. Un modèle représentatif donne une description statique de ce que connaît déjà le musicien, mais n'a que peu ou pas d'incidence directe sur son jeu, alors que le modèle opérationnel joue un rôle dynamique dans la maîtrise du déroulement de l'interprétation'. 
Dans la musique classique hindoustanie du Nord de l'Inde, la théorie musicale sert de modèle opérationnel. Aspect le plus manifeste de la théorie musicale, la notation revêt une importance capitale dans l'acquisition des compétences nécessaires pour jouer une musique réputée difficile qui requiert de nombreuses années d'apprentissage. Le fait de donner des noms aux notes et aux sons des tambours permet de les identifier, de les classer et de " constituer un continuum » (Harwood 1976). La notation orale est un moyen d'enseignement, un langage qui permet de véhiculer des informations. Les représentations verbales des hauteurs ou des sons des tambours servent de cadres cognitifs autour desquels s'organisent les séquences de mouvements des mains dans le jeu instrumental. Quant aux chanteurs, ils chantent aussi le nom des notes, dans certains passages improvisés. L'utilisation de la notation orale semble développer des capacités musicales très spécifiques qui permettent de passer du domaine verbal à celui des représentations aurales et motrices de la structure des compositions.

Pour moi, cette fonction opérationnelle n'est pas seulement déterminante dans l'apprentissage de la pratique musicale; elle joue aussi un rôle décisif dans le jeu des musiciens compétents. La notation orale et verbale devient un mode de pensée musicale. Ceux qui pratiquent ce système ont découvert comment exploiter le processus verbal dans la performance musicale. Ils peuvent, par exemple, mémoriser verbalement des compositions musicales et y accéder par le biais du processus de la pensée verbale. Au cours de l'improvisation, quand le musicien joue une chose et réfléchit en même temps à ce qui va suivre, cette anticipation peut dépendre en partie de la pensée verbale (Baily $1988 \mathrm{~b}: 118)$.

Il semble évident que, pour les musiciens de Hérat, la théorie musicale servait de modèle représentatif et non de modèle opérationnel. Un seul d'entre eux, cependant, faisait exception et semblait plus proche de la pratique hindoustanie, utilisant sa connaissance de la théorie musicale comme un modèle opérationnel. Il s'agit d'Ustad Amir Jan Khushnawaz, chanteur et joueur de rubâb, avec qui j'ai beaucoup travaillé dans les années 1970, et qui était un des rares musiciens de Hérat maîtrisant la notation sargam. Amir Jan avait acquis cette compétence auprès de son propre professeur, un ustâd de Kaboul, avec qui il avait étudié dans les années 1930. Cette formation lui avait appris l'art de désigner les hauteurs de façon précise et d'analyser les râg en fonction des notes et des échelles qui les constituaient. J'ai souvent eu l'occasion d'écouter avec lui des enregistrements de musique hindoustanie et de voir comment il procédait, même avec des râg qui lui étaient totalement inconnus. La notation sargam lui avait donné une oreille très analytique qui lui permettait de dicter des compositions avec des noms de notes, dits ou chantés. C'est ainsi que j'ai pu transcrire quantité de ses compositions. Dans l'ensemble, il préférait chanter les noms de notes à leurs hauteurs respectives plutôt que de les dire (Baily 1994 : 340). Il est évident que, pour Amir Jan, la théorie musicale servait de modèle opérationnel de la structure musicale plutôt que de modèle représentatif. Sa connaissance de la théorie musicale et sa capacité de l'utiliser de façon opérationnelle furent peut-être les facteurs principaux qui lui permirent d'établir et de maintenir son rôle prédominant au sein des musiciens de Hérat pendant plus de trente ans. (Baily 1988b : 120). 
Fig. 1 : Ustad Amir Jan Khushnawaz. Le meilleur spécialiste du rubâb de Hérat

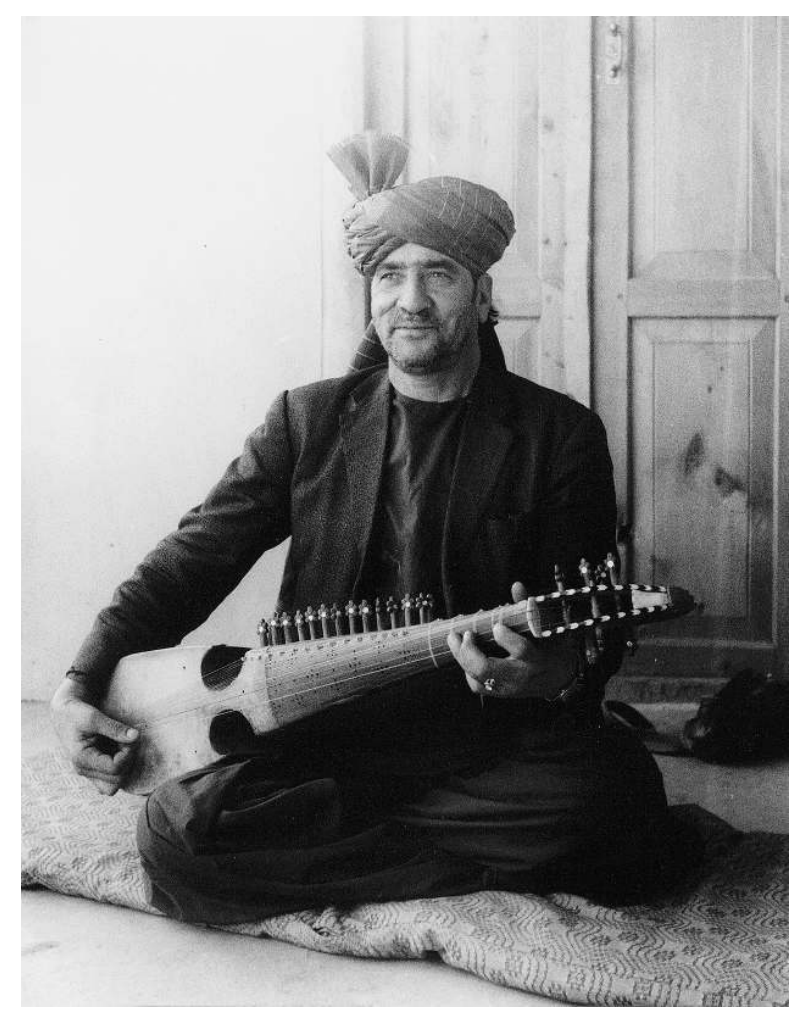

Photo : John Baily, 1977

\section{Connaissance semi-spécialisée}

11 On a beaucoup écrit, en ethnomusicologie, sur la distinction faite dans plusieurs régions d'Afghanistan, entre le statut d'amateurs et celui de professionnels, entre les shauqi et les kesbi (Slobin 1976, Sakata 1983, Baily 1988). Idéalement, tout au moins pour ce qui se disait à Hérat, le shauqi, terme appliqué uniquement aux hommes ${ }^{7}$, était une personne qui jouait par amour pour la musique et non pour gagner sa vie et qui refusait toute rémunération pour ses prestations musicales. N'étant pas issu d'une famille de musiciens professionnels, il était un autodidacte et affirmait n'avoir reçu l'enseignement d'aucun maître en particulier. Sa méthode d'apprentissage était celle de l'imitation. N'ayant pas été élevés dans des familles de musiciens professionnels et n'ayant pas reçu les encouragements d'un milieu où la musique constituait la préoccupation centrale (Doubleday and Baily 1995 : 442), les musiciens shauqi adultes ne souhaitaient d'habitude pas que leurs fils se consacrent à la musique. Ils faisaient même tout ce qui était en leur pouvoir pour les en décourager. Dans un tel contexte, les shauqi apprenaient leur métier secrètement, en ayant bien soin de cacher leur passion pour la musique à leur famille, afin d'éviter toute critique.

12 Les shauqi pouvaient devenir des musiciens très compétents mais ils avaient rarement accès à la science musicale, laquelle n'était pas enseignée dans les livres. La musique ne faisait pas partie du programme scolaire. Même si les musiciens amateurs devenaient d'excellents interprètes, leur connaissance de la science musicale restait généralement assez maigre, ils ne maîtrisaient pas la notation verbale, leurs connaissances des tâl et des 
râg étaient limitées, et ils auraient été en mal de parler des aspects techniques de la structure musicale. Ces lacunes n'échappaient pas aux professionnels (sâzandeh) qui traitaient les amateurs de shanidegi (ceux qui ont appris d'oreille), tandis qu'ils s'appelaient eux-mêmes darsei (ceux qui ont suivi un enseignement). On pourrait interpréter ces différences dans l'attribution du statut d'amateur ou de professionnel comme résultant d'une concurrence dans la profession musicale. Les musiciens héréditaires considéraient la musique comme leur sphère d'activité légitime et se méfiaient des aspirations des musiciens amateurs en voie de professionnalisation, à entrer en compétition avec eux.

Pourtant, bien que le shauqi fût un amateur au plein sens du terme, dès les années 1950, sous l'influence de Radio Afghanistan, quelques musiciens amateurs commencèrent à entrer dans la profession et à collaborer avec les musiciens héréditaires. A Hérat, le phénomène était perceptible surtout dans la pratique du dutâr, luth à long manche qui se transforma beaucoup entre les années 1950 et 1965. Instrument à deux cordes, destiné à l'origine à la musique rurale domestique, le dutâr finit par être pourvu de quatorze cordes dont plusieurs à fonction sympathique, permettant de jouer le répertoire de la musique savante kabouli, généralement réservée aux sâzandeh. Ces derniers ne jouaient jamais eux-mêmes du dutâr mais ils se mirent à intégrer des joueurs de dutâr dans leurs orchestres. Avec le temps, ceux-ci finirent par acquérir quelque connaissance de l'ilm-e musiqi. L'exemple classique de ce processus est peut-être celui du musicien hérati Karim Dutari, généralement connu comme l'inventeur du dutâr à quatorze cordes, ou du moins celui qui l'a perfectionné. Amateur à l'origine, il travailla pendant dix-sept ans à Radio Afghanistan comme joueur de dutâr. Parallèlement, il fut, à Kaboul, l'élève d'Ustad Mohammad Omar, à l'époque le plus éminent joueur de rubâb du pays. Tout en adaptant le dutâr à la musique savante de Kaboul, il acquit de nombreuses connaissances pratiques et théoriques de la musique indienne, devint avec le temps un spécialiste de la science musicale et joua par la suite un rôle important dans l'enseignement de la musique à Hérat. En 1974, il se lamentait de ce que ses étudiants préféraient apprendre en l'écoutant jouer alors qu'il aurait souhaité leur enseigner par l'intermédiaire de la notation. Un autre joueur de dutâr amateur, Gada Mohammad, devint un professionnel renommé. Durant de nombreuses années, il joua dans l'orchestre du chanteur kabouli Amir Mohammad, établi à Hérat. Au cours de cette longue collaboration, il apprit les détails d'un grand nombre de râg. Étant instruit, contrairement à la plupart des musiciens héréditaires, il était capable de transcrire les shakl, phrases caractéristiques de nombreux râg ${ }^{8}$. Il est évident que ce contact avec la science de la musique fut d'un grand secours dans sa carrière de musicien.

14 A Hérat, d'autres types de musiciens professionnels jettent également une lumière particulière sur cette question de la connaissance de la théorie musicale comme, par exemple, les ensembles de musiciennes professionnelles qui jouaient pour divertir les femmes lors des cérémonies de mariage. Elles n'avaient pratiquement aucune connaissance théorique de la musique qu'elles interprétaient. L'état de leurs connaissances était semblable à leurs instruments, d'habitude vieux et délabrés. Leurs tabla n'étaient plus susceptibles d'être accordés correctement et, de toute façon, ils étaient joués avec une technique de frappes à main plate évoquant plutôt le jeu du bongo. En comparant les musiciens amateurs aux professionnels, les hommes aux femmes, on remarque que la connaissance constituait une certaine forme de pouvoir, ou plutôt, que la 
répartition de la connaissance impliquait la répartition du pouvoir, du moins en ce qui concernait l'exécution musicale.

On pourrait faire des remarques analogues dans le cas des joueurs de sornâa (hautbois) et de dohol (tambour sur cadre à deux peaux), instruments joués à la campagne, à l'occasion des rites de passage, notamment les noces, par les barbiers-musiciens de classe inférieure et marginalisés. Certains d'entre eux avaient beau jouer avec une grande virtuosité, leur connaissance de la science musicale restait cependant très limitée.

\section{Connaissance non spécialisée}

16 Les non-musiciens étaient peu au fait des détails de l'ilm-e musiqi même s'ils n'en ignoraient pas l'existence. Ils avaient leur propre discours de non-spécialistes sur la musique. Je ne tiendrai pas compte ici des propos de ceux qui s'opposaient à la musique en la condamnant comme harâm ou satanique, car beaucoup a déjà été écrit à ce sujet ; je préfère attirer l'attention de l'observateur européen sur les aspects positifs de la culture islamique.

De nombreux Hératis percevaient une dimension spirituelle et positive dans la musique. Une des métaphores les plus courantes était de voir la musique comme une nourriture de l'âme, qazâye ruh. Les êtres humains avaient faim de musique comme le corps a faim d'aliments. Une variante de cette métaphore était que la musique rafraîchissait (tâzeh) l'âme. On entendait souvent l'expression musiqi del-e âdam zendeh mikone, «la musique vivifie notre cœur». Traduit par «cœur» bien que le cœur physique soit d'habitude appelé qalb, del était considéré comme le siège des sentiments, le centre émotionnel, le lieu de la conscience. La musique chassait l'ennui, soulageait la dépression et redonnait de la vitalité. Celui qui en jouait ne vieillissait jamais car elle gardait l'esprit jeune et alerte.

La musique avait la réputation de rendre heureux (khush), de procurer du plaisir (tahsir), de donner de la saveur (khub mazeh dâdan), d'être attirante (delchasp, littéralement « qui s'attache au del «). En l'écoutant, l'auditeur était transporté (lezat bordan). La musique pouvait être mélancolique (ghamgin), parfois même tragique (tragid, un anglicisme), ces deux termes servant surtout à décrire la musique classique persane. Un jeune joueur de dutâr, amateur issu d'une famille riche et très cultivé, m'a tenu un des propos les plus intéressants qu'il m'ait été donné d'entendre sur la musique :

«La musique est une sorte de langage qui dit quelque chose, mais de façon indirecte. Par la musique, les gens peuvent exprimer leurs sentiments, leur bonheur, leur tristesse, leurs problèmes et leurs différents états d'âme. La musique peut refléter ces sentiments » (Baily $1988: 153)$.

19 Une autre métaphore décrivant la musique était celle de l'eau. Une musique qui était bien jouée et donnait du plaisir était appelée târ, «humide ", alors que, dans le cas contraire, elle était khushk, « sèche ». Un compliment que l'on faisait habituellement en écoutant un morceau particulièrement attrayant était audâr, «juteux » (littéralement " qui contient de l'eau »). Les Hératis aimaient beaucoup la campagne, la nature, les jardins, les sites pittoresques agrémentés d'une belle vue, surtout au printemps. Le jardin représentait, dans leur littérature et leurs miniatures, un avant goût du paradis. Un jardin ombragé offrait le cadre idéal pour écouter un concert. 
Ils croyaient que la musique plaisait aux animaux comme aux humains et qu'elle nourrissait également leur âme. L'imagerie populaire de Hérat décrivait le musicien, d'habitude un joueur de dutâr assis dans un jardin ou dans un bel endroit, jouant si bien que les rossignols s'approchaient de lui et venaient parfois se percher sur les chevilles de son instrument. Les Hératis pensaient que les oiseaux étaient encouragés dans leurs chants lorsqu'ils entendaient la musique, aussi on apportait parfois des oiseaux en cage lors des réunions musicales. La symphonie qui en résultait, chants d'oiseaux mêlés de sons musicaux, constituait l'apogée du plaisir esthétique de la musique à Hérat (Baily 1997).

\section{Interaction entre connaissance spécialisée et non spécialisée}

21 Les discussions spécialisées et non spécialisées sur la musique ne se développaient pas dans des sphères complètement distinctes. Une certaine interaction existait entre elles. Les propos des non-spécialistes sur la musique étaient parfaitement familiers aux sâzandeh, mais le discours de ces derniers allait plus loin, était plus élaboré et plus détaillé. Dans une ville comme Hérat, on publiait peu sur la musique dans la presse populaire, il n'existait pas non plus de livres à ce sujet, les discussions sur la musique étaient rares à la radio, et plus tard à la télévision, qui se bornaient à diffuser la musique telle quelle, et il n'y avait bien entendu pas de musicologues. C'étaient les musiciens professionnels qui fournissaient en général ce genre d'informations au public. En d'autres termes, non seulement ils jouaient de la musique, mais ils produisaient un discours à son propos. Il est évident qu'une grande partie du discours non spécialisé provenait des sâzandeh eux-mêmes.

Fig. 2 : Amateurs de musique lors d'un mariage à Hérat

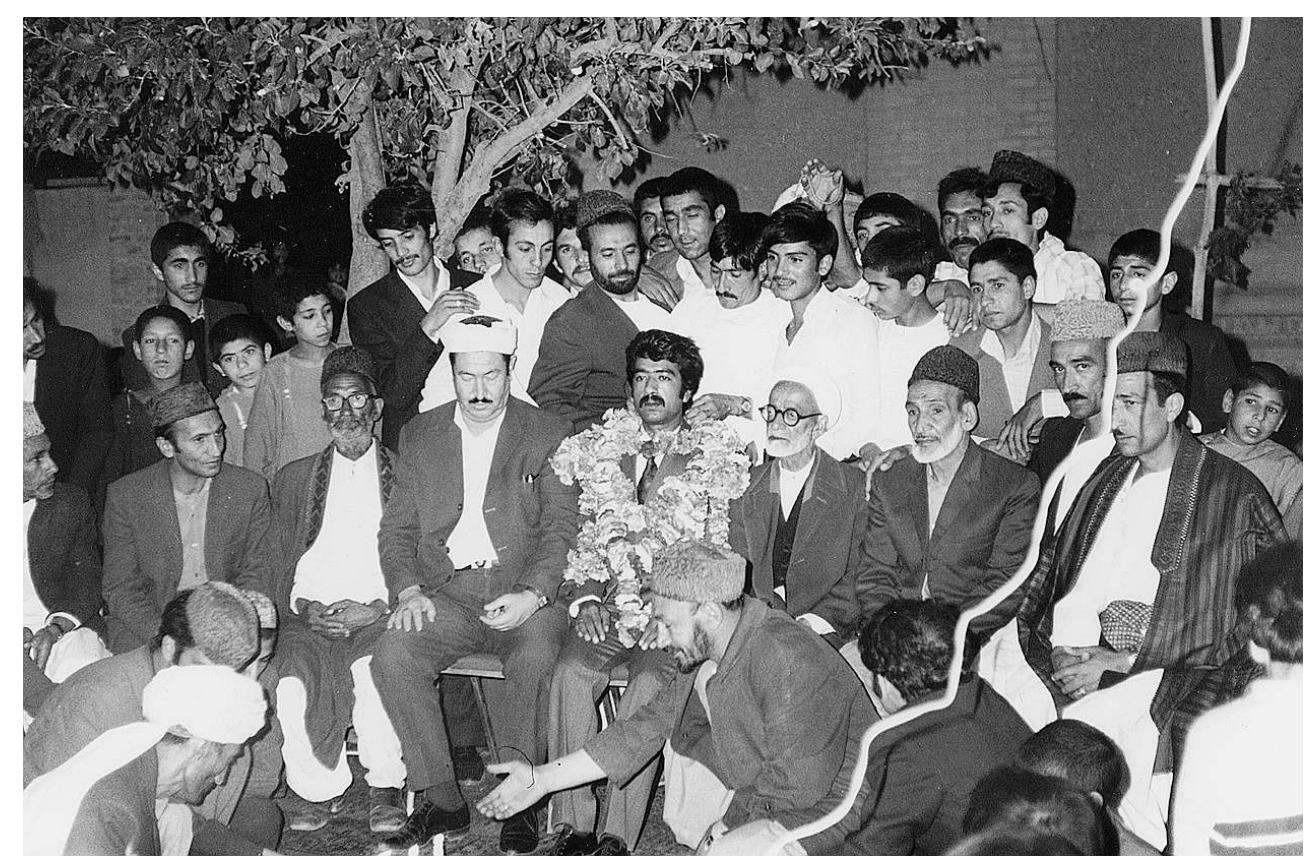

Photo: John Baily, 1974 
Les habitants de Hérat aimaient cancaner au sujet de la musique et des musiciens, surtout s'ils venaient d'ailleurs. Ils aimaient entendre des nouvelles du Kucheh Kharâbât, le quartier des musiciens de Kaboul (mais aussi celui des cabarets et des maisons closes), avec ses communautés de musiciens professionnels, dont certains étaient les descendants de musiciens de cour venus de Kasur au Pakistan ou de Patiala en Inde, tandis que d'autres étaient issus de familles de barbiers-musiciens. Ils voulaient connaître leurs origines, leurs relations, les scandales de leur vie personnelle, les querelles qui les opposaient et les orchestres dans lesquels ils avaient joué. Mais ils s'intéressaient peutêtre encore plus à la vie de certains musiciens amateurs comme Nashenas, qui obtint un doctorat en linguistique pashto de l'université du Moscou, ou Ahmad Zahir, fils d'un ancien premier ministre afghan, qui venaient l'un et l'autre du milieu bourgeois et cultivé et avaient choqué la bonne société en devenant des chanteurs populaires qui n'hésitaient pas à monter publiquement sur la scène musicale. Ailleurs dans le monde, ce genre de discussions sur la musique est entretenu par les médias ; à Hérat, c'est les musiciens euxmêmes qui se chargeaient de satisfaire la curiosité.

Un musicien tel que Ustad Amir Jan Khushnawaz tenait souvent ce genre de propos avec ses invités. Il donnait parfois des mehmâni, dîners auxquels il conviait ses amis et protecteurs pour leur offrir un repas somptueux, suivi d'un concert animé par lui-même et ses fils talentueux. Dans ces réceptions, la discussion tournait uniquement autour de la musique. Les invités regardaient les photos accrochées au mur, qui illustraient la carrière musicale de leur hôte et posaient des questions à leur sujet. Amir Jan racontait ses souvenirs et parlait de la musique et des musiciens de Kaboul et de l'Inde, décrivant ses propres séjours à Kaboul et ses rencontres avec les ustâd de cette ville, donnant et renforçant l'impression qu'il faisait partie de la scène des maîtres kabouli. C'était, en quelque sorte, l'artiste parlant de son œuvre.

Le joueur de dutâr Asiawan donne un bon exemple de ce genre de discours en racontant l'histoire du célèbre musicien amateur de Hérat, l'excentrique Amin-e Diwaneh, mort tragiquement au cours d'une bataille avec les pâluch («bandits») de Kandahar (Baily 1988a). Voici l'un des moments forts de son récit:

«Il y a beaucoup de jalousie dans le monde des joueurs de dutâr. Un jour, Karim Dutari, l'inventeur du dutâr à quatorze cordes, fut conduit en Jeep à Qasr-e Shirin, un village situé sur la route de Badghis, pour jouer de la musique dans une fête chez les Hazrats. Amin était également invité mais il s'y rendit à pied. Karim joua du dutâr à quatorze cordes. Tard dans la soirée, quelqu'un dit: "Donnons le dutâr à Amin et voyons ce qu'il est capable d'en faire ». Karim répliqua : "Amin ne peut pas toucher ce dutâr, il ne sait pas en jouer ». Furieux et bouleversé, Amin commença à se donner des coups et se taper la tête contre le mur, puis il demanda aux Hazrats : « Ne me laisserez-vous pas jouer?». Ses hôtes lui répondirent: «Oui, Diwaneh, prends le dutâr ! » Le musicien se mit alors à jouer tant et si bien que tout le monde fut émerveillé par son jeu et oublia Karim. Amin dit ensuite: «Avec votre permission, je vais couper les frettes de cet instrument et je jouerai sans frettes ", ce qu'il fit pour le plus grand plaisir des auditeurs. Puis, s'adressant à Karim, il dit : "Ceci est ton instrument, j'ai du nylon dans ma poche et je peux te renouer les frettes ; mais, si tu veux, tu peux jouer comme cela». Les invités s'écrièrent en chœur : « Non, tu dois continuer à jouer ». Et c'est ainsi qu'il joua jusqu'au matin. Le lendemain, on conduisit Amin en Jeep à Hérat, tandis que Karim dut revenir à pied, ne trouvant plus personne pour le ramener» (Baily 1988a : 141).

Un autre habile narrateur de la même veine était Amir Mohammad Hérawi, un musicien professionnel quelque peu marginalisé de Hérat, avec qui j’ai tourné le film Amir (Baily 
1985). C'était en 1977, nous nous étions retrouvés avec un petit groupe d'amis à l'occasion d'une sortie au site pittoresque de Mir Daud. Amin parla beaucoup, nous racontant entre autres l'histoire de Lal Shahbaz Qalandar, le célèbre musicien et saint sufi du Sind :

«Lal Shahbaz jouait du rubâb en compagnie de son disciple, quelque part dans la forêt. Un saint homme l'entendit de loin et s'approcha pour écouter sa musique. C'était un de ces fous (diwâneh) à cheveux longs et aux ongles non taillés, comme on en rencontre dans ce genre d'endroit. En ce temps là, le musicien ne s'appelait encore que Shahbaz Qalandar. L'ermite s'assit près d'eux et le musicien lui joua du rubâb pendant un long moment. Quand il eut fini, le saint homme pria : «Que Dieu transforme tes mains en rubis ", le rubis (lal) signifiant quelque chose d'unique et de très précieux. Avec l'aide de Dieu, ses mains rougirent soudain et se mirent à émettre une lumière incandescente, tandis que ses doigts devenaient translucides, comme du rubis. Ravi, Lal Shahbaz Qalandar remercia le saint et lui parla avec gentillesse. Son disciple lui dit: "Maintenant que vous avez des mains de rubis, vous devriez aller en Inde et être prêt à donner votre tête pour la cause d'une musique aussi parfaite. » Lal Shahbaz Qalandar répondit: " Bien sûr, je suis prêt. » En ce temps là, il y avait de nombreux mullahs en Inde qui n'aimaient pas la musique, ils la détestaient même. Lal Shahbaz Qalandar se rendit donc en Inde. Un jour qu'il jouait du rubâb dans une maison, quelqu'un l'entendit et rapporta la chose à l'âkhund (mullah). "Qu'on me l'amène ! » ordonna celui-ci. On fit comparaître le musicien et lui demanda ce qu'il faisait. "Ceci est ma passion (shauq) », leur avoua$\mathrm{t}$-il, «c'est mon pir (maître spirituel) qui m'a dit de jouer». Les mullahs se demandèrent ce qu'ils devaient faire de lui et décidèrent finalement de le décapiter. Il fut emmené sur la place publique tandis que le crieur ameutait la foule en hurlant : « Il jouait de la musique ! C'est interdit ! On va le décapiter ! ॥ Un mullah se leva, prit son épée et, sans autre forme de procès, lui trancha la tête, la détachant complètement du corps. Alors, avec la bénédiction de Dieu, les cheveux de la tête coupée se mirent à produire de la musique. De chaque cheveu sortit un air différent: en Bairami, Kesturi, Bihag, Jog, Asawari, Multani, Delbari, Ahir Beyru, Yemen, Sham Kalyan. Les mullahs furent sidérés : «Que s'était-il passé ?» A partir de ce moment, la musique fut peu à peu acceptée en Inde et ce flux musical finit par atteindre un niveau tel qu'aujourd'hui la musique indienne est reconnue comme une des plus belles du monde ${ }^{9}$ ».

Le récit que donne Amir Jan Hérawi de la mort de Lal Shahbaz Qalandar nous ramène au point de départ de cet article, les mullahs et leur propre science de la musique, «la science de la prononciation correcte du Coran ". Il soulève aussi une autre question, celle des origines de la musique classique indienne. En effet, beaucoup d'Afghans prétendent que celle-ci a pris naissance en Afghanistan, dans la ville de Balkh, et qu'elle a été introduite en Inde au XIII ${ }^{\mathrm{e}}$ siècle par Amir Khusro Balkhi, un grand lettré des cours du Sultanat de Delhi. La légende lui attribue de nombreuses innovations dans la musique hindoustanie, telles que l'invention du sitâr et des tabla et la création des genres vocaux khyâl et qawwâli. D'après les Afghans, la musique a prospéré dans le climat de tolérance religieuse qui régnait en Inde, alors qu'en Afghanistan, les mullahs entravaient son développement. Plus récemment, la musique hindoustanie a retrouvé son lieu véritable d'origine. Ce genre de prétentions cadrait bien avec le discours nationaliste apparu en Afghanistan dans les année 1930, qui soutenait que le pashto était la langue aryenne la plus ancienne et la plus pure et que «l'Avesta et les premiers Védas étaient les plus grands chefs d'œuvre de la littérature afghane et pashto » (Gregorian 1969 : 347) ${ }^{10}$. 


\section{Conclusions}

Les faits énoncés plus haut montrent qu'il existait plusieurs types de discours sur la musique à Hérat. D'une part, la connaissance de la théorie musicale, réservée aux musiciens professionnels urbains. D'autre part le discours sur l'histoire de la musique, la biographie des musiciens célèbres et les idées sur la place occupée par la musique dans la vie humaine, que l'on pourrait appeler philosophie de la musique. Il n'est pas surprenant que des gens qui étaient arrivés à la musique par des voies différentes aient tenu à son propos des discours différents et qu'il ait existé entre eux des rapports hiérarchiques. Ainsi le discours des non-spécialistes pouvait être tenu par les spécialistes mais le contraire n'était pas possible. La théorie musicale était essentiellement la compétence des musiciens professionnels, elle faisait partie de leur connaissance spécialisée et de leur art traditionnel. L'essentiel du discours des non-spécialistes provenait probablement de celui des spécialistes. Dans le discours de ces derniers, on pouvait distinguer les modèles de structures musicales ayant un rôle représentatif ou opérationnel.

Dans toute société, on trouve probablement des personnes qui ont une connaissance approfondie. Dans le cas de Hérat, j'ai rencontré deux spécialistes, Amir Jan et Maulawi Nurahmad, qui travaillaient dans deux « sphères sonores » très différentes, la musique et la récitation coranique. Il n'est pas sans intérêt de constater que, dans les deux cas, ils avaient acquis leur connaissance à l'extérieur : Amir Jan étudia l'ilm e musiqi auprès de l' ustâd Nabi Gol dans les années 1930, lorsque ce musicien de Kaboul fit plusieurs séjours prolongés à Hérat, et Maulawi Nurahmad avait appris l'ilm-e tajwid alors qu'il étudiait la théologie en Inde. Ces personnes correspondent peut-être à Irisipau, le spécialiste local, ami d'Hugo Zemp (Hugo Zemp 1978, 1979). L'ethnomusicologue avide d'informations détaillées à propos de la théorie musicale doit rechercher ces spécialistes locaux.

Ce qui semblait manquer, dans une société comme celle d'Hérat, était le rôle joué par les musicologues et les critiques, rôle qui va de soi en Occident. Le musicologue est une sorte d'« amateur professionnel », un étudiant de la musique qui peut fort bien jouer, mais qui ne dépend pas de cette pratique pour vivre. Le critique est payé pour parler de musique dans les livres, les revues, les magazines, les journaux, à la radio et à la télévision. La société occidentale a énormément à dire sur la musique, nonobstant le «dilemme de Seeger" (Herndon 1974: 244). Les habitants de Hérat aimaient entendre parler de musique mais ils disposaient de peu de ressources et avaient des moyens d'information limités. Dans une telle société, les musiciens spécialisés fournissaient non seulement la musique, mais aussi un discours à son propos, à une vaste audience de non-spécialistes.

\section{BIBLIOGRAPHIE}

BAILY John, 1985, Amir: An Afghan Refugee Musician's life in Peshawar, Pakistan. Film de16mm. Version vidéo disponible auprès du Royal Anthropological Institute, Londres. 
BAILY John, 1988, Music of Afghanistan: Professional musicians in the city of Herat. Cambridge: Cambridge University Press. Accompagné d'une cassette audio.

BAILY John, 1988a, « Amin-e Diwaneh: The musician as madman », Popular Music 7(2): 131-146.

BAILY John, 1988b, « Anthropological and psychological approaches to the study of music theory and musical cognition », Yearbook for Traditional Music, 20: 114-124.

BAILY John, 1994, « Learning to perform as a research technique in ethnomusicology », in: Chung Kyo-Chul and Uwe Pätzold (ed.) Lux Oriente - Encounters of Cultures in Scientific Musical Research. A Feschrift for Robert Günther, University of Cologne: 331-347.

BAILY John, 1997, « Afghan Perceptions of Birdsong », The World of Music: 39(2): 51-59.

BAILY John, et Veronica DOUBLEDAY, 1988, « Modèles d'imprégnation musicale en Afghanistan », Cahiers de musiques traditionnelles 1. De bouche à oreille : 112-124.

BELIAEV V.M., 1960, Abdurrakhman Dzhami : « Traktat omusyke ». Tashkent: Izd-vo ANUzSSR

DOUBLEDAY, Veronica, and John BAILY, 1995, « Patterns of musical development among children in Afghanistan ", in Elizabeth Fernea (ed), Children in the Muslim Middle East, Austin: University of Texas Press: 431-444.

ENGLISH Paul, 1973, "The traditional city of Herat, Afghanistan », in L. Carl Brown (ed.), From Madina to Metropolis: Heritage and Change in the Near Eastern City. Princeton: The Darwin Press: 73-90.

FELD Steven, 1981, «'Flow Like a Waterfall': The Metaphors of Kaluli Music Theory », Yearbook of Traditional Music XIII: 22-47.

GREGORIAN Vartan, 1969, The Emergence of Modern Afghanistan. Stanford: California University Press.

HARWOOD Dane, 1976, « Universals in Music: A Perspective from Cognitive Psychology », Ethnomusicology 22: 521-533

HERNDON Marcia, 1974, « Analysis: Herding of Sacred Cows? », Ethnomusicology 18: 219-262.

MERRIAM Alan P., 1964, The Anthropology of Music. Evanston, Ill.: Northwestern University Press.

SAKATA Hiromi Lorraine, 1983, Music in the Mind. The Concepts of Music and Musician in Afghanistan. Kent : Kent State University Press. Accompagné de deux cassettes audio.

SCHIMMEL Annemarie, 1975, Mystical Dimensions of Islam. Chapel Hill: University of North Carolina Press.

SLOBIN Mark, 1976, Music in the Culture of Northern Afghanistan. Tucson: University of Arizona Press. (Viking Fund Publications in Anthropology $\mathrm{N}^{\circ} 54$ ).

ZEMP Hugo, 1978, « 'Are'are classification of musical types and instruments », Ethnomusicology 22: 37-68.

ZEMP Hugo, 1979, « Aspects of 'Are'are musical theory » Ethnomusicology 23: 5-48.

\section{NOTES}

2. L'ancienne cité de Hérat se trouve à l'Ouest de l'Afghanistan, à quelque 125 kilomètres de la frontière iranienne. English (1963) a fait une description utile de la ville à la fin des années 1960. La plupart des recherches qui ont servi à la rédaction de cet article ont été menées par l'auteur, 
de 1973 à 1977, et complétées par des contacts prolongés avec les musiciens afghans résidant au Pakistan, en Europe, en Amérique du Nord et en Afghanistan, depuis 1977. Au moment de la rédaction (1998), les instruments de musique et la musique sont complètement bannis dans les deux tiers de l'Afghanistan, y compris à Hérat et à Kaboul, actuellement sous contrôle des Talibans. C'est pour cette raison que j'ai choisi d'utiliser le passé qui doit représenter l'état probable du discours sur la musique à Hérat aujourd'hui, bien que je sois certain qu'il contribue toujours à animer les discussions des Hératis de la diaspora.

3. Il peut sembler étrange de parler de «notation orale " pour les noms de notes et les syllabes de tambour alors que notre conception habituelle de la notation implique l'existence de symboles écrits ou imprimés sur du papier ou tout autre support. Toutefois, ces noms de notes et ces syllabes mnémoniques exprimés verbalement servent bel et bien de notation. Ils sont les équivalents fonctionnels des unités sonores qui permettent de créer les modèles musicaux et aident à l'analyse aussi bien qu'à la mémorisation. L'expression «notation orale » cherche à rendre cette idée.

4. D'après Feld, les Kaluli utilisaient une théorie musicale pour l'enseignement et l'apprentissage, quand ils passaient de l'expression verbale au chant. Ils transmettaient systématiquement la théorie musicale comme un savoir et l'utilisaient avec Feld pour analyser les exemples de chants enregistrés ou pour critiquer ses propres essais de composition de chants kaluli. Ils s'en servaient également pour parler des musiques qui ne leur étaient pas familières comme les enregistrements du saxophoniste américain John Coltrane que Feld leur soumettait.

5. Le terme «imitation" peut paraître ambigu quand il est appliqué aux processus d'imprégnation musicale. L'imitation peut faire partie de ce que Merriam (1964, chapitre 8) a appelé «éducation » et "instruction », comme par exemple lorsque le professeur montre une courte phrase et que l'élève doit la répéter. Mais le terme «imitation » est employé ici dans un sens différent pour décrire un processus d'apprentissage indirect où l'enfant essaie de reproduire spontanément quelque chose qu'il vient de voir et d'entendre, selon un processus autoguidé et autodidacte (Doubleday and Baily 1995 : 441-443).

6. La théorie occidentale de la musique, avec sa notation sur portée, ses systèmes de clefs et son harmonie fonctionnelle tonale, est manifestement l'exemple d'un modèle opérationnel de structure musicale.

7. On peut se demander pourquoi le terme shauqi désignait si rarement les femmes. A mon avis, un tel abîme séparait, dans ce cas précis, les professionnels des amateurs qu'il n'était même pas nécessaire d'évoquer cette distinction. Il était évident pour tout le monde que les femmes ordinaires qui pouvaient chanter et jouer le tambour sur cadre appartenaient à une catégorie complètement différente des rares femmes issues de familles de musiciens héréditaires qui gagnaient leur vie en jouant de la musique pour divertir les femmes, lors des cérémonies de mariage.

8. Shakl désigne la section introductive de la partie instrumentale d'un chant, dans laquelle les passages improvisés en rythme libre sont joués dans le cadre des contraintes mélodiques du mode à exécuter.

9. Le mot persan lal désigne un joyau, le rubis. Je remercie Nabi Misdaq de m'avoir aidé à traduire le récit quelque peu embrouillé d'Amir que j'ai tenté de réorganiser ici. Son histoire de Lal Shahbaz est apparemment complètement fantaisiste. Selon Annemarie Schimmel, Lal Shahbaz, dont le nom signifie "le faucon rouge", était un saint de Sehwan, dans le Sind. Mystique extatique, son sanctuaire a attiré d'étranges groupes de soufis « affranchis des lois » (Schimmel 1975 : 355).

10. Ces idées sur la musique et la religion ont acquis une nouvelle dimension depuis la fin du régime communiste en Afghanistan, en 1992. Avec le gouvernement Rabani, la musique était certainement sous contrôle sévère bien que pas entièrement condamnée, alors que, depuis l'arrivée des Talibans, elle a été complètement bannie. 


\section{RÉSUMÉS}

Trois types de discours musicaux ayant cours dans la ville afghane de Hérat dans les années 1970 sont analysés ici: le discours des spécialistes, celui des semi-spécialistes et celui des nonspécialistes. Il existe une relation hiérarchique entre ces trois discours dans la mesure où les spécialistes peuvent tenir le discours des non-spécialistes alors que le contraire n'est pas possible. Le discours des spécialistes, appelé «science de la musique ", est une théorie musicale héritée du sous-continent indien. A l'intérieur de ce discours, on distingue en outre les modèles représentatifs des modèles opérationnels de la structure musicale, selon le rôle que joue la connaissance exprimée verbalement au cours de la performance musicale. Le modèle représentatif est une rationalisation a posteriori des pratiques musicales acquises par le processus imitatif, tandis que le modèle opérationnel intervient directement aussi bien dans l'apprentissage de la musique que dans son interprétation par des musiciens chevronnés.

Three kinds of musical discourse about music as practised in the Afghan city of Herat in the 1970s are examined: those of experts, semi-experts, and non-experts. There is a hierarchical relationship between these, in that experts may engage in the discourse of non-experts, but not vice versa. The discourse of experts is couched in terms of the "science of music", a music theory derived from the Indian sub-Continent. A further distinction is made within expert discourse between representational and operational models of music structure, depending on the role or knowledge which can be expressed verbally in the actual performance of music. The representational model is a post hoc rationalisation of performance practices established by imitative procedures, while the operational model is one which mediates both directly in the acquisition of musical skills and in performance by the skilled practitioner.

\section{AUTEURS}

\section{JOHN BAILY}

John Baily est ethnomusicologue et cinéaste. Dès 1978, il enseigne l'ethnomusicologie, la musique et le cinéma dans plusieurs universités du Royaume-Uni ainsi qu'aux Etats-Unis. Il occupe actuellement la chaire d'ethnomusicologie au Goldsmiths College de l'Université de Londres. Auteur de nombreuses publications, d'enregistrements et de films sur la musique afghane et les musiciens de Hérat, il joue lui-même du rubâb kabouli et du dutâr hérati. 